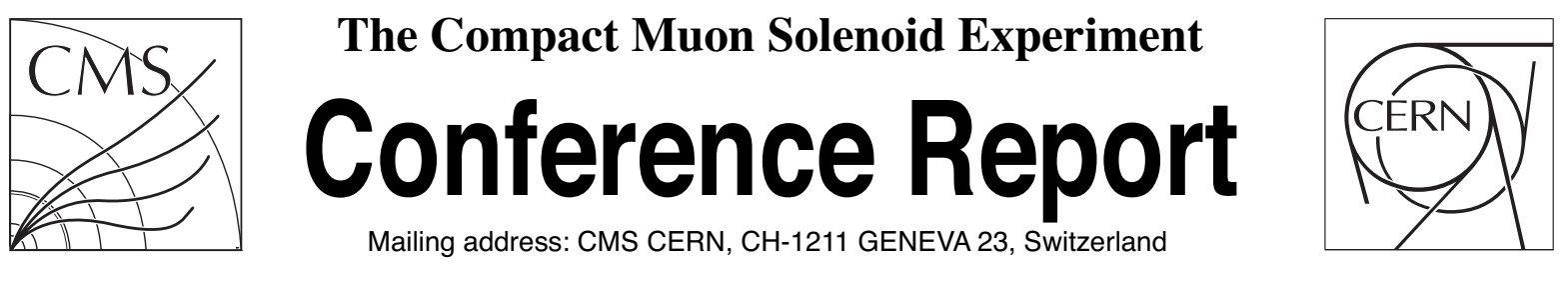

01 July 2015 (v2, 01 August 2015)

\title{
Design and performance of the upgrade of the CMS L1 muon trigger
}

Pierluigi Bortignon for the CMS Collaboration

\begin{abstract}
After the Long Shutdown 1 (LS1) LHC will run at a center of mass energy of $13 \mathrm{TeV}$, providing CMS with proton collisions at an expected luminosity which is almost double the LHC design value of $10^{34} \mathrm{~cm}^{-2} \mathrm{~s}^{-1}$, and almost three times the peak luminosity reached during Run 1 of $7.7 \cdot 10^{33} \mathrm{~cm}^{-2} \mathrm{~s}^{-1}$. The higher luminosity and center of mass energy of the LHC will raise the Level 1 (L1) muon trigger rate by almost a factor six for a given muon transverse momentum $\mathrm{p}_{\mathrm{T}}$ threshold. It is therefore necessary to increase the muon $\left(\mathrm{p}_{\mathrm{T}}\right)$ threshold to keep the trigger rate below $100 \mathrm{kHz}$, the maximum sustainable rate for the CMS detectors. An increase of the L1 trigger thresholds implies a lowering of the efficiency in detecting signals from new physics. The CMS muon trigger is upgraded using custom designed AMC boards, with more powerful FPGAs and larger memories. The upgraded CMS muon trigger system implements pattern recognition and MVA (Boosted Decision Tree) regression techniques in the trigger boards for muon $\mathrm{p}_{\mathrm{T}}$ assignment, drastically reducing the trigger rate and improving the trigger efficiency. The upgraded system design exploits the redundancy of the CMS muon detectors at a very early stage merging different muon detector information already at L1. The pileup subtracted information from the upgraded calorimeter trigger allows to require isolated muons already in the L1 algorithms. The upgrade trigger is also designed to include inputs from GEM, the phase 2 muon detector upgrade in the very high pseudorapidity region.
\end{abstract}




\title{
Design and performance of the upgrade of the CMS L1 muon trigger
}

\author{
P. Bortignon ${ }^{\mathrm{a}}$, on behalf of the CMS collaboration \\ ${ }^{a}$ University of Florida, Gainesville, USA
}

\begin{abstract}
After the Long Shutdown 1 (LS1) LHC will run at a center of mass energy of $13 \mathrm{TeV}$, providing CMS with proton collisions at an expected luminosity which is almost double the LHC design value of $10^{34} \mathrm{~cm}^{-2} \mathrm{~s}^{-1}$, and almost three times the peak luminosity reached during Run1 of $7.7 \cdot 10^{33} \mathrm{~cm}^{-2} \mathrm{~s}^{-1}$. The higher luminosity and center of mass energy of the LHC will raise the Level 1 (L1) muon trigger rate by almost a factor six for a given muon transverse momentum $\mathrm{p}_{\mathrm{T}}$ threshold. It is therefore necessary to increase the muon $\left(\mathrm{p}_{\mathrm{T}}\right)$ threshold to keep the trigger rate below $100 \mathrm{kHz}$, the maximum sustainable rate for the CMS detectors. An increase of the L1 trigger thresholds implies a lowering of the efficiency in detecting signals from new physics. The CMS muon trigger is upgraded using custom designed AMC boards, with more powerful FPGAs and larger memories. The upgraded CMS muon trigger system implements pattern recognition and MVA (Boosted Decision Tree) regression techniques in the trigger boards for muon $\mathrm{p}_{\mathrm{T}}$ assignment, drastically reducing the trigger rate and improving the trigger efficiency. The upgraded system design exploits the redundancy of the CMS muon detectors at a very early stage merging different muon detector information already at L1. The pileup subtracted information from the upgraded calorimeter trigger allows to require isolated muons already in the L1 algorithms. The upgrade trigger is also designed to include inputs from GEM, the phase 2 muon detector upgrade in the very high pseudorapidity region.
\end{abstract}

Keywords: Front end, Trigger, DAQ and Data Management EM $\mu \mathrm{TF}$

\section{The CMS muon trigger design}

The upgraded L1 CMS muon trigger [1] consists of four subsystems: three regional track finders and one global muon trigger. The three track finders cover three different pseudorapidity regions and receive trigger primitives from different muon detector technologies. All track finders send their trigger primitives to the global muon trigger which removes duplicates across track finder boundaries and ranks the muon trigger primitives before sending them to the CMS global trigger.

\subsection{Barrel Muon Track Finder}

The barrel muon track finder receives all hits produced by the CMS muon chambers installed in the central region of the detector up to pseudorapidity $|\eta|<0.8$. These include Drift Tubes (DT) and Resistive Plate Chambers (RPC). Optical links connect the front end electronics to the track finder boards. The Barrel Track Finder custom boards called MP7 are based on a Virtex7 FPGA. These new FPGAs reduce the latency of the barrel track finder, and, in a second stage, allow the development of more sophisticated algorithms to improve the muon $\mathrm{p}_{\mathrm{T}}$ assignment performance.

\subsection{Endcap Muon Track Finder}

The endcap muon track finder triggers events with muons leaving signals in the CMS encdap detectors: the Cathode Strip Chambers (CSC) and the RPC in that region. It uses MTF7 boards, $\mu$ TCA boards specifically designed to measure the muon $\mathrm{p}_{\mathrm{T}}$ with the best precision within the latency allocated by CMS for that purpose $(\approx 750 \mathrm{~ns})$. It covers the forward region of CMS, with $1.25<|\eta|<2.5$. Optical signals are sent from the CSC and the RPC to the trigger boards that use a large memory to load a Look Up Table (LUT) to transform patterns of hits in a $\mathrm{p}_{\mathrm{T}}$ measurement. The design of the EM $\mu \mathrm{TF}$ boards allows to accept GEM detector inputs in vision of the installations of this new type of muon chambers [2].

\subsection{Overlap Muon Track Finder}

The experience of LHC Run1 showed that the muon trigger system has special needs in the region where the magnetic field is not uniform. The overlap track finder takes care of this complex region included between $0.8<|\eta|<1.25$. It uses the same board design as the endcap muon track finder (MTF7) but with a customised firmware and algorithm for $\mathrm{p}_{\mathrm{T}}$ assignment. The overlap track finder receives input from all three muon detectors installed in CMS and combines their information in order to reach the best muon $\mathrm{p}_{\mathrm{T}}$ assignment.

\subsection{Global Muon Trigger}

The global muon trigger combines and ranks all muons sent by the three regional systems (barrel, overlap, and endcap track finder) according to their $\mathrm{p}_{\mathrm{T}}$ measurement accuracy. Then the highest ranked muons are sent to the Global Trigger, the final step of the L1 triggering system of CMS. Similar to the other trigger systems, the global muon trigger upgraded its copper links to optical links, and it will receive many more muon candidates than during Run1. It is using CPT7 boards which will allow a better handling of a higher number of trigger primitives. 




Figure 1: Comparison of the Run1 trigger system (CSCTF) trigger rate on a single muon gun monte carlo sample with the upgrade system (EM $\mu \mathrm{TF})$ with different thresholds for tail clipping.

\section{Physics Performance}

New possible physics phenomena will become within reach with the upgrade in energy and intensity of the LHC. It is crucial to keep the triggering system at least as efficient as it was for Run1. Physics analyses which will benefit from the muon trigger upgrade are Higgs boson searches like in WH production with $\mathrm{H} \rightarrow \mathrm{b} \bar{b}$ and $\mathrm{H} \rightarrow$ WW with both $\mathrm{W}$ decaying leptonically, but also supersymmetry analyses like the search for direct Stop quarks production. During Run1 these analyses were using a Single Muon trigger to collect their signal. With the LHC expected Run2 luminosity this trigger will produce $27 \mathrm{kHz}$, a trigger rate that would use a too high bandwidth in the CMS L1 trigger system. The upgraded system is reducing this rate for the same muon $\mathrm{p}_{\mathrm{T}}$ threshold while keeping the signal efficiency high.

\section{Tail clipping}

A tail clipping method reduces the rate without affecting the signal efficiency. Tail clipping is a post-processing step that allows to route the LUT output back to the main FPGA. For a given tail fraction a boundary curve is computed for every variable. Every muon candidate with a $\mathrm{p}_{\mathrm{T}}$ evaluated from the LUT that lies outside this boundary is demoted to the maximum allowed value. This procedure is repeated for all variables and eventually the minimum $\mathrm{p}_{\mathrm{T}}$ found using this procedure overwrites the $\mathrm{p}_{\mathrm{T}}$ from the LUT. Figure 1 shows, for different tail fractions, the rate reduction obtained by this procedure on an endcap muon track finder emulation.

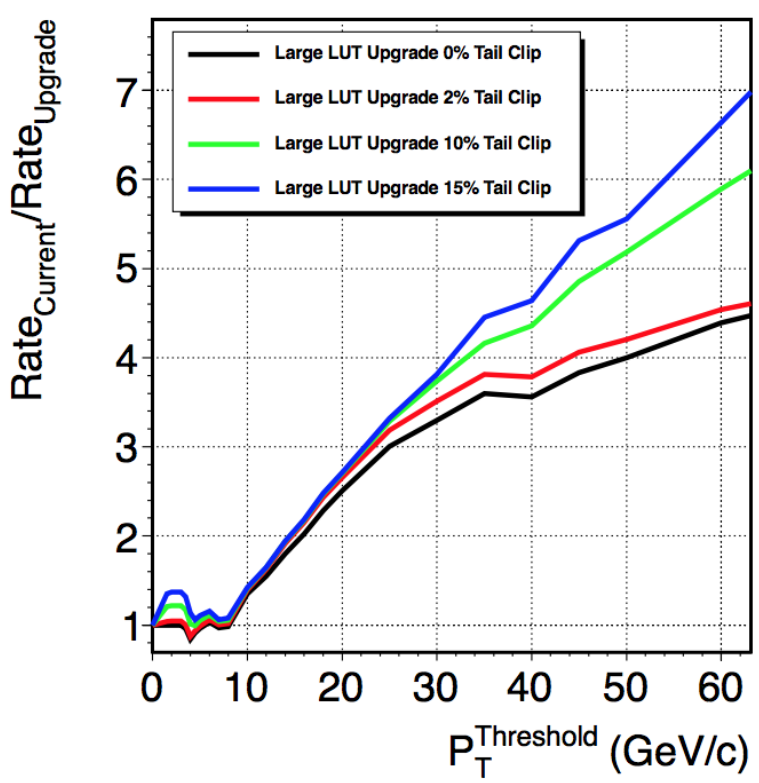

Figure 2: Comparison the Run1 trigger system (CSCTF) trigger rate on a single muon gun monte carlo sample with the upgrade system (EM $\mu \mathrm{TF})$ which here uses a Boosted Decision Tree to assign the $p_{\mathrm{T}}$ to the muons. The rate is compared for multiple thresholds of tail clipping.

\section{FPGA with Multi Variate Analysis for $p_{\mathrm{T}}$ assignment}

A LUT is created based on the response of a Boosted Decision Tree (BDT) trained on Monte Carlo events: the BDT takes as input sensitive information of the muon candidates and it estimates the muon transverse momentum using a regression analysis. The resolution in $\mathrm{p}_{\mathrm{T}}$ increases compared to the Maximum Likelihood method used during Run1. It reduces the muon $\mathrm{p}_{\mathrm{T}}$ mis-assignment probability and therefore the muon rate at low $\mathrm{p}_{\mathrm{T}}$. Figure 2 shows the ration between the rate of the Run1 endcap muon trigger (current) and the rate of the upgraded endcap muon trigger using large LUT with a BDT based muon $\mathrm{p}_{\mathrm{T}}$ assignment. The improvement is notable for muons of $\mathrm{p}_{\mathrm{T}}>8 \mathrm{GeV}$.

\section{Conclusion}

A new, better performing, and easier to maintain level1 muon trigger is being installed in CMS and is ready to be tested and commissioned using a parasitic running on the legacy trigger system with the new data arriving from the LHC Run2 at 13 $\mathrm{TeV}$. The final switch to the the upgraded muon trigger is foreseen after its full commissioning is completed at the beginning of 2016.

\section{References}

[1] CMS collaboration: CMS Technical Design Report for the Level-1 Trigger Upgrade. CERN-LHCC-2013-011. CMS-TDR-12

[2] A. Colaleo, and A. Safonov, and A. Sharma, and M. Tytgat: CMS technical design report for the muon endcap GEN upgrade. CERN-LHCC2015-012. CMS-TDR-013 